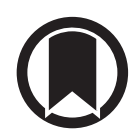

CrossMark

\title{
A potential therapeutic role for angiotensin-converting enzyme 2 in human pulmonary arterial hypertension
}

\author{
Anna R. Hemnes ${ }^{1,6}$, Anandharajan Rathinasabapathy ${ }^{1,6}$, Eric A. Austin², \\ Evan L. Brittain ${ }^{3}$, Erica J. Carrier ${ }^{1}$, Xinping Chen ${ }^{1}$, Joshua P. Fessel ${ }^{1}$, \\ Candice D. Fike ${ }^{2}$, Peter Fong ${ }^{3}$, Niki Fortune ${ }^{1}$, Robert E. Gerszten ${ }^{4}$, \\ Jennifer A. Johnson ${ }^{1}$, Mark Kaplowitz ${ }^{2}$, John H. Newman ${ }^{1}$, Robert Piana ${ }^{3}$, \\ Meredith E. Pugh ${ }^{1}$, Todd W. Rice ${ }^{1}$, Ivan M. Robbins ${ }^{1}$, Lisa Wheeler ${ }^{1}$, Chang Yư ${ }^{5}$, \\ James E. Loyd ${ }^{1}$ and James West ${ }^{1}$
}

Affiliations: ${ }^{1}$ Division of Allergy, Pulmonary and Critical Care Medicine, Vanderbilt University Medical Center, Nashville, TN, USA. ${ }^{2}$ Dept of Pediatrics, Vanderbilt University Medical Center, Nashville, TN, USA. ${ }^{3}$ Division of Cardiovascular Medicine, Vanderbilt University Medical Center, Nashville, TN, USA. "Division of Cardiovascular Medicine, Beth Israel Deaconess Medical Center, Boston, MA, USA. ${ }^{5}$ Dept of Biostatistics, Vanderbilt University Medical Center, Nashville, TN, USA. ${ }^{6}$ These two authors are joint first authors.

Correspondence: Anna R. Hemnes, Division of Allergy, Pulmonary and Critical Care Medicine, Vanderbilt University Medical Center, T1218 MCN, 1161 21st Avenue South, Nashville, TN 37212, USA.

E-mail: anna.r.hemnesवvanderbilt.edu

@ERSpublications

The ACE2-Ang-(1-7)-Mas1 axis is probably involved in the pathophysiology of human pulmonary arterial hypertension http://ow.ly/pgS530jOxnd

Cite this article as: Hemnes AR, Rathinasabapathy A, Austin EA, et al. A potential therapeutic role for angiotensin-converting enzyme 2 in human pulmonary arterial hypertension. Eur Respir J 2018; 51: 1702638 [https://doi.org/10.1183/13993003.02638-2017].

ABSTRACT Pulmonary arterial hypertension (PAH) is a deadly disease with no cure. Alternate conversion of angiotensin II (AngII) to angiotensin-(1-7) (Ang-(1-7)) by angiotensin-converting enzyme 2 (ACE2) resulting in Mas receptor (Mas1) activation improves rodent models of PAH. Effects of recombinant human (rh) ACE2 in human $\mathrm{PAH}$ are unknown. Our objective was to determine the effects of rhACE2 in PAH.

We defined the molecular effects of Masl activation using porcine pulmonary arteries, measured AngII/ Ang-(1-7) levels in human PAH and conducted a phase IIa, open-label pilot study of a single infusion of rhACE2 (GSK2586881, 0.2 or $0.4 \mathrm{mg} \cdot \mathrm{kg}^{-1}$ intravenously).

Superoxide dismutase 2 (SOD2) and inflammatory gene expression were identified as markers of Mas1 activation. After confirming reduced plasma ACE2 activity in human $\mathrm{PAH}$, five patients were enrolled in the trial. GSK2586881 was well tolerated with significant improvement in cardiac output and pulmonary vascular resistance. GSK2586881 infusion was associated with reduced plasma markers of inflammation within 2-4 h and increased SOD2 plasma protein at 2 weeks.

PAH is characterised by reduced ACE2 activity. Augmentation of ACE2 in a pilot study was well tolerated, associated with improved pulmonary haemodynamics and reduced markers of oxidant and inflammatory mediators. Targeting this pathway may be beneficial in human PAH.

This article has supplementary material available from erj.ersjournals.com

This study is registered at clinicaltrials.gov with identifier number NCT01884051.

Received: Dec 182017 | Accepted after revision: April 252018

Copyright OERS 2018 


\section{Introduction}

Pulmonary arterial hypertension (PAH) is a highly morbid disease that primarily affects young females with progressive pulmonary vascular obliteration resulting in right heart failure and death. While much progress has been made in improving outcomes in $\mathrm{PAH}$, therapies are imperfect and there is presently no cure. Newer, more effective treatments are needed that address key disrupted pathways in PAH.

Although the renin-angiotensin-aldosterone system is known to be activated in PAH [1-4], trials of angiotensin-converting enzyme (ACE) inhibitors in this disease have not demonstrated benefit [5-8], and although angiotensin II (AngII) type $1\left(\mathrm{AT}_{1}\right)$ receptor antagonism has benefit in the monocrotaline rat model of pulmonary hypertension, this drug class has not been trialled in humans with PAH [1]. The observation has led to the hypothesis that alternate hydrolysis of AngII to angiotensin-(1-7) (Ang-(1-7)) via ACE2 may be a more effective therapeutic intervention. Ang-(1-7) activates the Mas receptor (Mas1), which is present on endothelial cells, and has vasodilatory, anti-inflammatory and antifibrotic effects [9, 10], functionally antagonising the effects of $\mathrm{AT}_{1}$ receptor stimulation [11-13]. Finally, activation of the ACE2-Ang-(1-7) axis reduces oxidant stress in diabetes mellitus [14], suggesting impact on pathways of relevance to $\mathrm{PAH}$ [15-17]. Thus, the ACE2-Ang-(1-7)-Mas1 axis may be a promising therapeutic pathway in $\mathrm{PAH}$.

We and others have demonstrated that both infusion of ACE2 and direct activation of Mas1 ameliorate rodent models of PAH [18-23], likely through improved cytoskeletal function, which is consistent with prior work on ACE2 [24]. Furthermore, the ACE2-Ang-(1-7) axis has been studied in a right ventricular failure model in which ACE2 peptide administration resulted in reduced right ventricular hypertrophy and fibrosis with improved function [19], suggesting potentially beneficial effects on both the pulmonary vasculature and also right ventricular load stress responses. In human patients, there are no US Food and Drug Administration-approved mechanisms to stimulate Mas1 and there are no direct Mas1 agonists approved for human use. ACE2 enzymatic activity can be augmented by administration of an intravenous formulation of the soluble recombinant human form of the naturally occurring enzyme (rhACE2) with existing safety data in healthy volunteers and acute respiratory distress syndrome (GSK2586881; ClinicalTrials.gov identifier NCT01597635) [25].

We tested the hypotheses that ACE2 activity is reduced in human PAH compared with healthy controls and that short-term ACE2 administration may be safe in a proof-of-concept pilot study of GSK2586881 in PAH patients. We further sought to identify short-term markers of Mas1 activation, suggesting molecular drug effect, which may facilitate future studies of Mas1 activation in $\mathrm{PAH}$.

\section{Methods}

Animal experiments

All animal studies were approved by the Institutional Animal Care and Use Committee of Vanderbilt University Medical Center (VUMC), Nashville, TN, USA.

\section{Pulmonary arterial isolation and cannulation}

Pulmonary arteries (80-300 $\mu \mathrm{m}$ diameter) were dissected from portions of piglet lungs using previously described methods [26]. RNA sequencing experiments were performed as previously reported [27, 28]. See supplementary material for full details.

\section{Observational studies of ACE2 in PAH patients}

Human studies were approved by the VUMC Institutional Review Board (numbers 9401 and 151388) and registered at ClinicalTrials.gov (identifier NCT01884051). All patients gave written informed consent prior to inclusion in the study. Idiopathic and heritable $\mathrm{PAH}$ patients, aged $\geqslant 18$ years, defined according to standard criteria by expert clinicians, were included [29]. See supplementary material for details of reninangiotensin system (RAS) peptide measurement and aptamer-based superoxide dismutase 2 (SOD2) protein measurement.

\section{Pilot trial of GSK2586881}

The study was a phase I dose-escalation, open-label proof-of-concept study in patients with idiopathic or heritable PAH, functional class I-III. The primary end-point was safety, with secondary end-points of change in disease biomarkers (brain natriuretic peptide, AngII/Ang-(1-7) ratio, SOD2 activity and nitrotyrosine activity), and systemic and pulmonary haemodynamics and echocardiographic metrics of $\mathrm{PAH}$ including right ventricular function. We planned three patients at $0.2 \mathrm{mg} \cdot \mathrm{kg}^{-1}$ based on prior safety data with an escalation to $0.4 \mathrm{mg} \cdot \mathrm{kg}^{-1}$ if no dose-limiting toxicity occurred. See supplementary material for detailed inclusion and exclusion criteria. 
Patients were recruited from the pulmonary hypertension clinic at VUMC from March 2016 to December 2016. Study procedures are outlined in supplementary table S3. Briefly, patients underwent right heart catheterisation. Haemodynamics were recorded $1 \mathrm{~h}$ prior to drug administration $(-1 \mathrm{~h})$, immediately prior to drug administration $(0 \mathrm{~h})$, and 1,2 and $4 \mathrm{~h}$ after drug administration. Patients were observed overnight with the 6-min walk test (6MWT) and echocardiography measured $24 \mathrm{~h}$ after drug administration. Patients returned 2 weeks after drug administration for safety assessment, 6MWT and clinical evaluation. Safety end-points assessed included change in functional class, development of right heart failure signs or symptoms, 6MWT distance, plasma electrolytes and markers of renal function, complete blood count, and echocardiography-derived right ventricular function.

See supplementary material for details of RAS peptide measurement, SOD2 ELISA, nitrotyrosine dot-blot assay, cytokine Luminex assay, and isoprostane and isofuran measurement

\section{Statistical analysis}

Continuous variables of demographic data are reported as mean with standard deviation. The Wilcoxon rank-sum test and the Mann-Whitney U-test were used to compare differences between groups. Categorical variables were compared between groups using the Chi-squared test or Fisher's exact test. A p-value of $<0.05$ was considered statistically significant. Data from the pilot study were compared using the paired two-tailed t-test. Statistical analyses were performed using Prism version 5.0 (GraphPad, La Jolla, CA, USA) and R version 3.0.1 (www.r-project.org).

\section{Results}

\section{ACE2 activity in human PAH}

We first tested the hypothesis that ACE2 activity is suppressed in human PAH patients compared with controls. We enrolled 11 consecutive heritable or idiopathic PAH patients and eight healthy controls (two males and six females) (supplementary table S1). We measured AngII and Ang-(1-7) in plasma, and found a significant decrease in ACE2 activity as reflected by the AngII/Ang-(1-7) ratio in PAH compared with controls $(\mathrm{p}=0.01)$ (figure $1 \mathrm{a}-\mathrm{c})$. This difference was driven by an increase in plasma AngII, which was nearly four-fold higher in PAH than controls $(\mathrm{p}<0.003)$, with a less significant difference in Ang(1-7) levels in PAH versus controls.

\section{Biochemical effects of Mas1 activation}

We next sought to define the molecular consequences of Mas1 activation. As ACE2 requires AngII as substrate and Ang-(1-7) has a short half-life, the use of ACE2 in isolated artery studies is challenging. Subsequently we used AVE0991, a direct Mas1 agonist, to determine if Mas1 activation results in pulmonary artery vasodilation (figure 2a). Using AVE0991 in porcine pulmonary arteries pre-constricted with endothelin-1 [30], we found a dose-dependent increase in percentage dilation as a function of AVE0991, demonstrating that Mas1 activation has similar physiological effects in this model as seen in rodent models [18, 20, 21].

To determine the acute changes in gene expression as a result of Mas1 activation in this isolated artery model, we isolated RNA from arteries exposed to AVE0991 and controls, and performed RNA sequencing. Significantly changed genes are presented in supplementary table S2. Using a gene ontology analysis approach (figure $2 \mathrm{~b}$ ), we found significant changes in several pathways, including genes associated with pressure regulation $\left(\mathrm{p}<10^{-5}\right)$, inflammatory responses $\left(\mathrm{p}=10^{-4}\right)$, and leukocyte and cell migration $\left(\mathrm{p}<10^{-4}\right)$, again suggesting potential effects of Mas1 activation on inflammation and cytoskeletal function. In order to define a potential marker for Mas1 activation in the pulmonary artery, we compared pulmonary arterial gene expression levels before and after AVE0991 (figure 2c). Several genes were upregulated as a result of AVE0991 exposure, including TGIF1 (transforming growth factor- $\beta$-induced factor homeobox 1), CCL2 (C-C motif chemokine ligand 2) and SOD2. CCL2 is known to be important in $\mathrm{PAH}$, suggesting potential relevance of this research to the field [31]. SOD2, a mitochondrial protein catalysing production of hydrogen peroxide and oxygen from superoxide, was an attractive marker based on prior publications [32-34], and a known effect of Mas1 activation in reducing oxidative stress in rodent models of diabetes [14], suggesting potential biological plausibility and also its significant upregulation after AVE0991 exposure. Validation using quantitative PCR in pulmonary arteries can be found in supplementary figure S1. Furthermore, SOD2 expression was high in the control pulmonary arteries and demonstrated marked increase in gene expression after Mas1 activation, suggesting it may be a biologically important and sensitive marker of increased ACE2 activity.

\section{SOD2 in human PAH}

We next sought to determine if SOD2 levels were different in human PAH and control patients. We used an aptamer-based proteomic profiling approach to measure relative plasma SOD2 protein content in 

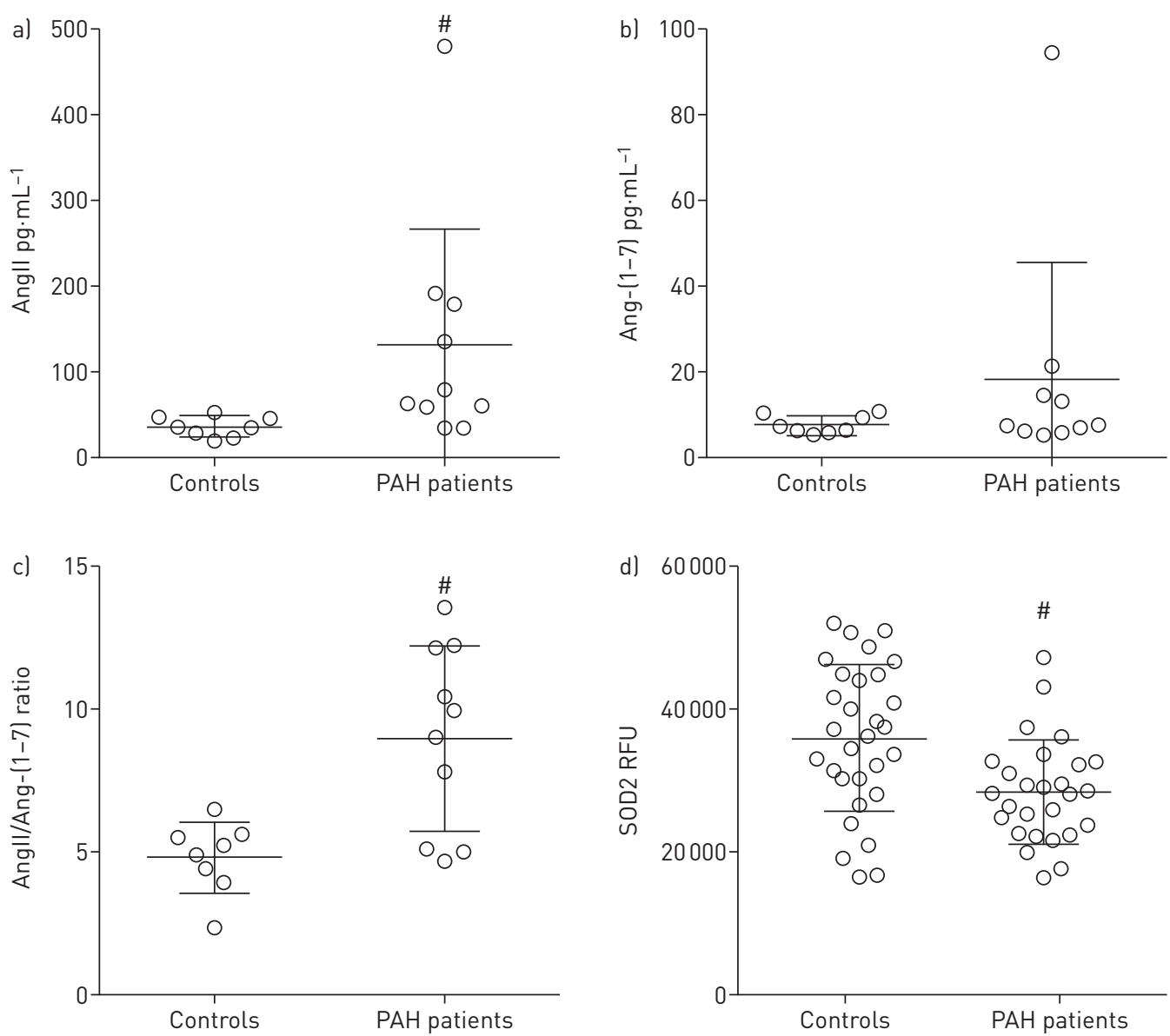

FIGURE 1 Evidence of insufficient angiotensin-converting enzyme 2 (ACE2) activity in human pulmonary arterial hypertension (PAH). a-c) Plasma Angll and Ang-(1-7) levels were measured in 10 PAH patients and eight healthy controls as a marker of ACE2 activity. Log-transformed values are normally distributed by the Shapiro-Wilk W-test for a) Angll, b) Ang-(1-7) and c) Angll/Ang-(1-7) ratio, allowing parametric testing. a) Angll was increased in PAH (\#: $p<0.003)$ and c) Angll/Ang-(1-7) ratio was increased in PAH (\#: $p=0.01)$, suggesting reduced conversion of Angll to Ang-(1-7) by ACE2 in the plasma of PAH patients. d) SOD2 protein level in plasma was measured by aptamer-based proteomic assay in $30 \mathrm{PAH}$ patients and 27 age-, sex- and body mass index-matched controls. SOD2 protein was reduced in $\mathrm{PAH}$. \#: $\mathrm{p}=0.002$. Angll: angiotensin II; Ang-(1-7): angiotensin-(1-7); SOD2: superoxide dismutase 2; RFU: relative fluorescence units.

fasting peripheral blood in $30 \mathrm{PAH}$ patients (age 49.5 \pm 13.2 years, body mass index (BMI) $29.8 \pm 8.2 \mathrm{~kg} \cdot \mathrm{m}^{-2}$, three males) and 27 matched controls (age $46.7 \pm 11.8$ years, BMI $29.1 \pm 6.7 \mathrm{~kg} \cdot \mathrm{m}^{-2}$, five males) (figure $1 \mathrm{~d}$ ). We found levels of SOD2 protein were $~ 25 \%$ lower in PAH plasma compared with controls $(28489 \pm 7112$ versus $35930 \pm 10202$ relative fluorescence units; $\mathrm{p}=0.002$ ).

\section{Pilot trial of GSK2586881 in PAH}

We next sought to determine the safety and potential acute haemodynamic and biochemical effects of a single i.v. infusion of GSK2586881 administration in human PAH patients (see supplementary table S3 for details of the protocol). We enrolled five PAH patients and used two different GSK2586881 doses (patients 1-3, $0.2 \mathrm{mg} \cdot \mathrm{kg}^{-1}$; patients 4 and 5, $0.4 \mathrm{mg} \cdot \mathrm{kg}^{-1}$ ) (table 1). All patients were receiving at least two $\mathrm{PAH}$-directed therapies including prostaglandin pathway treatments. All patients completed the study protocol and there were no serious adverse effects with study drug administration. One patient experienced dizziness without change in vital signs (patient 1 ) within $1 \mathrm{~h}$ of drug administration and one patient reported leg tingling during the overnight observation period (patient 5) that did not require intervention.

There were no statistical or clinically significant changes in other safety parameters, including 6MWT distance (supplementary table S4). All patients were functional class II at baseline and four were functional class II at the 2-week follow-up, one patient was functional class I. With the exception of a small but statistically significant decrease in creatinine from baseline to $24 \mathrm{~h}\left(1.03 \pm 0.4\right.$ versus $0.99 \pm 0.4 \mathrm{mg} \cdot \mathrm{dL}^{-1}$; 

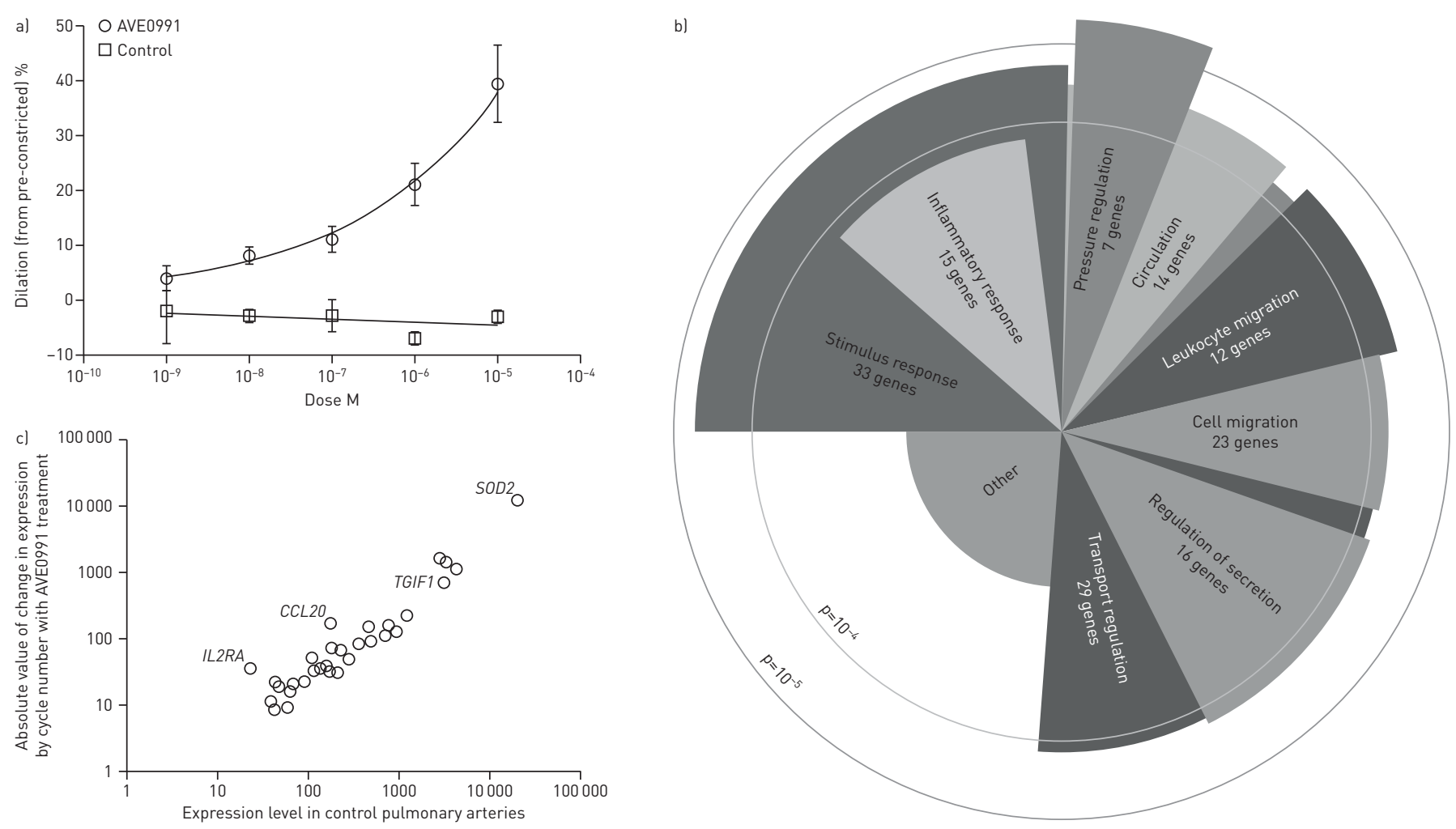

FIGURE 2 Acute molecular effects of Mas receptor (Mas1) activation in a porcine pulmonary hypertension model. a) AVE0991, a direct Mas1 agonist, was administered to pig arteries pre-constricted with endothelin-1. There was a dose-dependent increase in percentage dilation with AVE0991 administration. No dilation was seen with exposure to a biologically irrelevant control compound. b) RNA was isolated from pig arteries with and without AVE0991 exposure, and RNA sequencing was performed. Gene ontology analysis demonstrated significant differences in gene expression in several pathways, including inflammatory responses $\left(p=10^{-4}\right)$, cell and leukocyte migration $\left(p<10^{-4}\right)$, and pressure regulation $\left(p<10^{-5}\right)$. c) When absolute change in gene expression with drug exposure was plotted as dependent on expression level in control arteries, SOD2 expression was strongly upregulated in response to Mas1 activation. $\mathrm{n}=8$ vessels.

$\mathrm{p}=0.03$ ), there were no significant changes in laboratory values (data not shown). There were no differences in echocardiographic metrics of left ventricular or right ventricular function at $24 \mathrm{~h}$ (supplementary table S5). No antidrug antibodies were detected at 2 weeks in any patient (data not shown). Pharmacokinetic profiles are listed in supplementary table S6.

A single dose of GSK2586881 had no effect on mean pulmonary arterial pressure (supplementary table S7 and figure 3a); however, we did observe a statistically significant increase in cardiac output (5.3 \pm 1.4 versus $\left.6.1 \pm 1.3 \mathrm{~L} \cdot \mathrm{min}^{-1} ; \mathrm{p}=0.008\right) 4 \mathrm{~h}$ after study drug administration, representing an average increase of $40 \%$ (figure 3b). Pulmonary vascular resistance (PVR) would be expected to decrease when cardiac output increases without a change in mean pulmonary arterial pressure. We observed substantial variability in PVR with low numbers of enrollees, but when comparing combined time-points prior to drug

TABLE 1 Characteristics of patients enrolled in the GSK2586881 trial

\begin{tabular}{|c|c|c|c|c|c|c|c|}
\hline Patient & $\begin{array}{c}\text { Age } \\
\text { years }\end{array}$ & Sex & $\begin{array}{c}\text { BMI } \\
\mathrm{kg} \cdot \mathrm{m}^{-2}\end{array}$ & PAH type & $\begin{array}{c}\text { Disease } \\
\text { duration } \\
\text { months }\end{array}$ & Current therapy & Comorbid disease \\
\hline
\end{tabular}

\begin{tabular}{|c|c|c|c|c|c|c|c|}
\hline 1 & 28 & Female & 27.5 & Idiopathic & 13 & PP, PDE-5I & None \\
\hline 2 & 39 & Female & 27.8 & Idiopathic & 60 & PP, PDE-5I, ERA & None \\
\hline 3 & 57 & Male & 32.5 & Idiopathic & 91 & Selexipag, PDE-5I & DM2, systemic hypertension, hyperlipidaemia \\
\hline 4 & 41 & Female & 27.4 & Heritable & 92 & Inhaled treprostinil, PDE-5I, ERA & None \\
\hline
\end{tabular}

BMI: body mass index; PAH: pulmonary arterial hypertension; PP: parenteral prostaglandin; PDE-5I: phosphodiesterase type 5 inhibitor; ERA: endothelin receptor antagonist; DM2: type 2 diabetes mellitus. Patients 1,2 and 3 received $0.2 \mathrm{mg} \cdot \mathrm{kg}^{-1}$ GSK2586881; patients 4 and 5 received $0.4 \mathrm{mg} \cdot \mathrm{kg}^{-1}$ GSK2586881. 


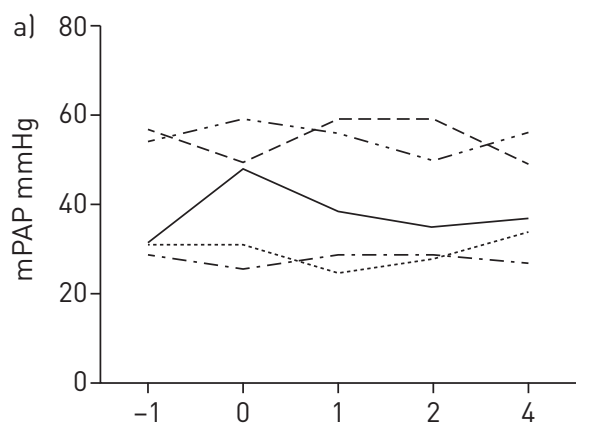

Time after drug administration $\mathrm{h}$

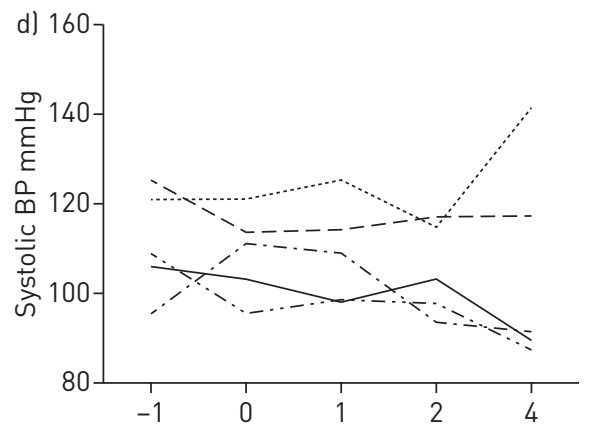

Time after drug administration $\mathrm{h}$

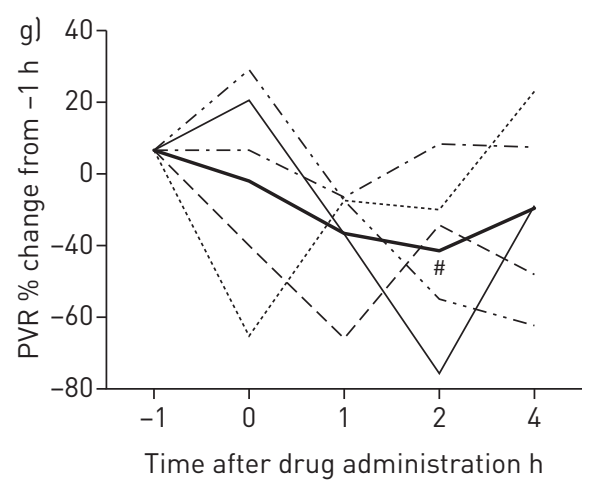

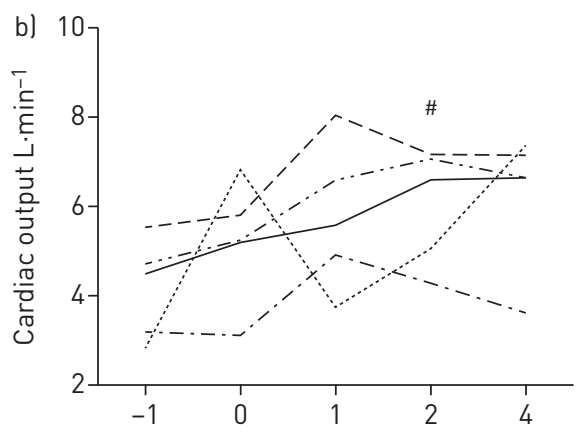

Time after drug administration $\mathrm{h}$
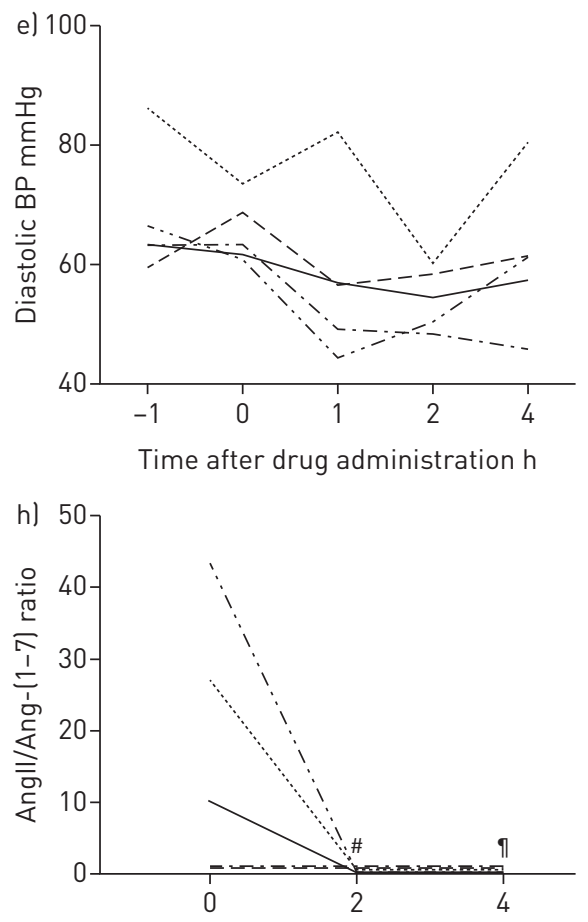

Time after drug administration $\mathrm{h}$

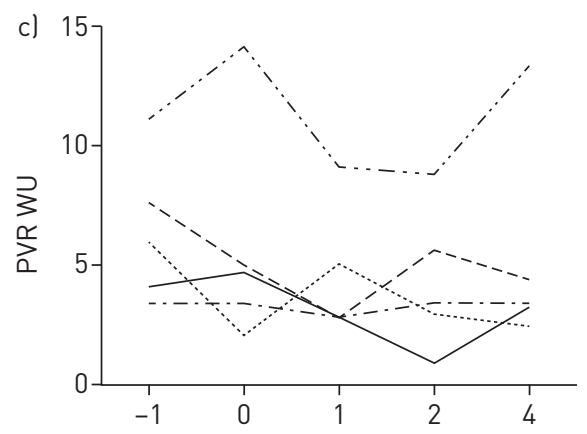

Time after drug administration $\mathrm{h}$

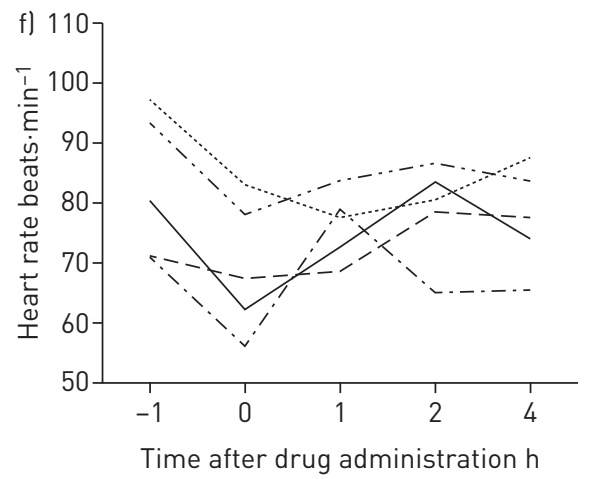

FIGURE 3 Pulmonary and systemic haemodynamic effects of intravenous GSK2586881. A pulmonary artery catheter was placed and haemodynamics measured ( $-1 \mathrm{~h}$ ). Just prior to GSK2586881 exposure, pulmonary haemodynamics were recorded again (0 h), and then at 1,2 and $4 \mathrm{~h}$ after drug administration. $\mathrm{n}=5$. a) There was no significant change in mPAP. b) There was a significant increase in cardiac output $2 \mathrm{~h}$ after drug administration. ${ }^{\#}: \mathrm{p}=0.008 . \mathrm{c}-\mathrm{f}$ ) Individual raw data on $\mathrm{c}$ ) PVR, d) systolic BP, e) diastolic BP and f) heart rate. g) Percentage change in PVR showed a significant decrease when comparing time-points before drug administration $(-1$ and $0 \mathrm{~h})$ with those after drug administration $(1,2$ and $4 \mathrm{~h}) .{ }^{\#}$ : $\mathrm{p}<0.05$. h) Angll and Ang-(1-7) levels were measured just prior to drug administration (0 h), and 2 and $4 \mathrm{~h}$ after drug adminstration. In the three patients with elevation in the Angll/Ang-(1-7) ratio at baseline, there was a significant decrease in the ratio, suggesting effective angiotensin-converting enzyme 2 augmentation with administration of GSK2586881. " $: p=0.009$ and ${ }^{\natural}: p=0.008$ versus 0 at 2 and 4 h, respectively. mPAP: mean pulmonary arterial pressure; PVR: pulmonary vascular resistance; WU: Wood unit; BP: blood pressure; Angll: angiotensin II; Ang-(1-7): angiotensin-(1-7).

administration with after infusion in normalised PVR, we found a statistically significant decrease in PVR, reaching a nadir $2 \mathrm{~h}$ after administration $(\mathrm{p}<0.05)$ (figure $3 \mathrm{~g}$ ). There were no significant effects on systemic haemodynamics (figure $3 \mathrm{~d}-\mathrm{f}$ ).

Plasma AngII and Ang-(1-7) levels were measured as a marker of clinical pharmacology. A decrease in the AngII/Ang-(1-7) ratio suggests increased activity of ACE2 and thereby effective augmentation by the study drug. There was significant variability in the AngII/Ang-(1-7) ratio at baseline (figure $3 \mathrm{~h}$ ). Three patients had substantial elevations in this ratio at baseline and, in these patients, there was a reduction to a nearly undetectable ratio with drug administration at $2 \mathrm{~h}(26.5 \pm 16.5$ versus $0.2 \pm 0.2 ; \mathrm{p}=0.009)$ that was sustained at $4 \mathrm{~h}(0.3 \pm 0.2 ; \mathrm{p}=0.008)$. Full details of changes in RAS peptides are in shown in supplementary figure S3.

Effect of GSK2586881 on SOD2 in human PAH

As our ex vivo animal model had shown induction of SOD2 expression with Mas1 activation, we tested the hypothesis that SOD2 protein content in PAH patient plasma would be induced by GSK2586881 
administration (figure 4a). Using an ELISA assay, we found that there was significant induction of plasma SOD2 protein levels by 2 weeks ( $\mathrm{p}=0.009$ versus baseline values) suggesting induction in the enzymatic activity by GSK2586881, corroborating our findings in the animal model and indicating that this may be a marker of Mas1 activation in human PAH.

\section{Effects of GSK2586881 on plasma markers of inflammation}

Our animal microarray data further suggested that Mas1 activation potentially acts through altering the pattern of pro-inflammatory gene expression. We next tested the hypothesis that this improvement would occur in human PAH patients after acute GSK2586881 administration. As markers of reduced pro-inflammatory signalling, we measured a plasma cytokine array and markers of oxidant stress. We utilised a Luminex platform to assess the effect of GSK2586881 on plasma cytokines (table 2). Consistent with prior reports [35], there were increased levels in six out of nine measured cytokines at time-point $0 \mathrm{~h}$ compared with controls $(\mathrm{p}<0.05$ for interleukin (IL)-10, IL-1 $\beta$, tumour necrosis factor (TNF)- $\alpha$, IL-13, IL-8 and IL-4). With GSK2586881 administration, there was suppression of measured cytokines including IL-10, IL-1 $\beta$, IL-2 and TNF- $\alpha$ that could be detected as early as $2 \mathrm{~h}$ after drug administration, and was
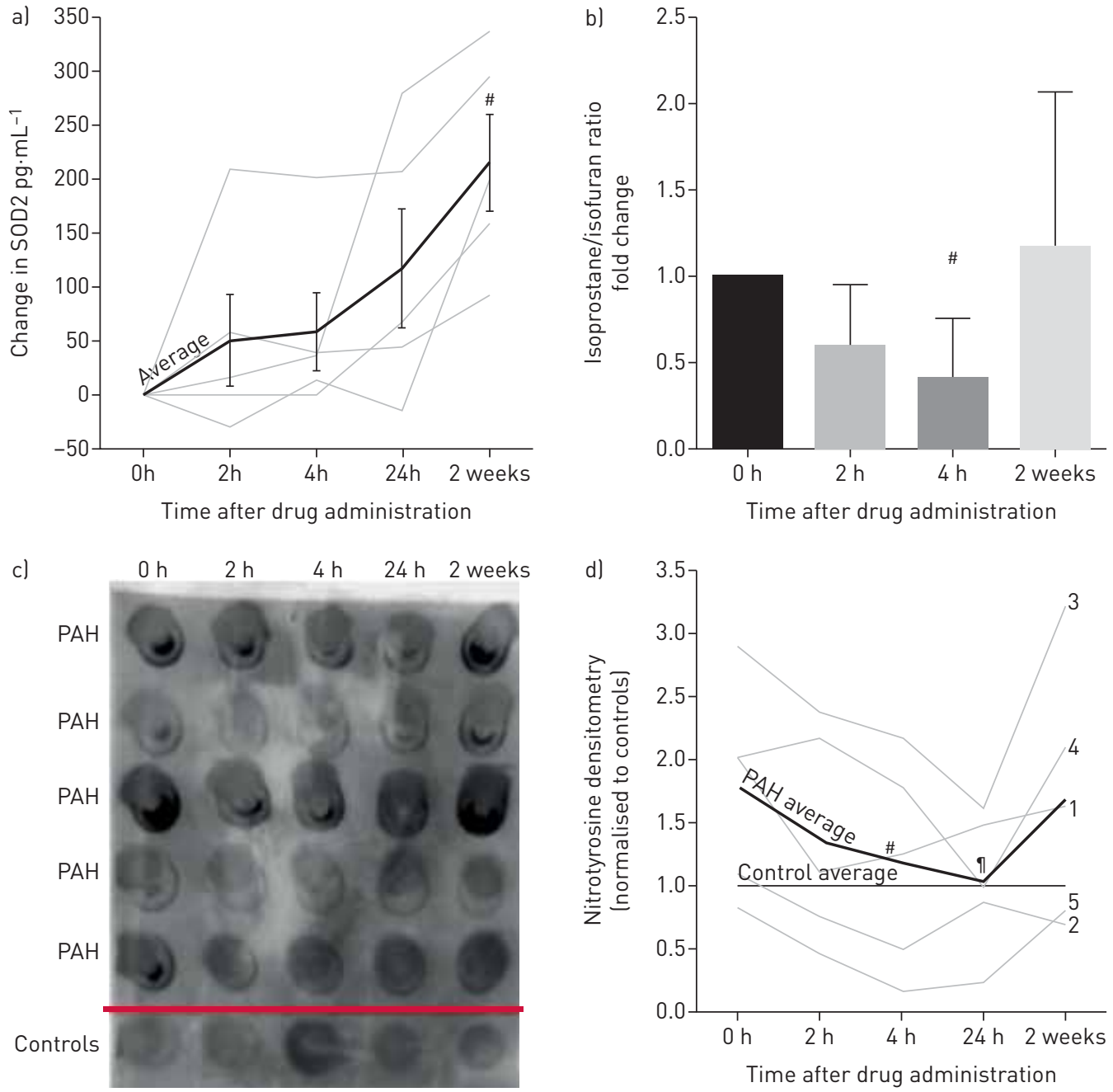

FIGURE 4 Effect of GSK2586881 on markers of oxidant stress. a) Plasma SOD2 protein levels were measured by ELISA assay just prior to drug administration $(0 \mathrm{~h})$, and 2,4 and $24 \mathrm{~h}$, and 2 weeks after drug administration. $n=5$. There was a significant increase in plasma SOD2 levels at 2 weeks. ${ }^{\#}: p=0.009$. b) We measured plasma isoprostanes and isofurans as markers of oxidant stress. Isofurans are lipid peroxidation products similar to isoprostanes, but are formed in times of oxygen availability. There was a decrease in the isoprostane/isofuran ratio, suggesting improved oxygenation. ${ }^{\#}: p<0.05$. c) Nitrotyrosine dot-blot assay from the plasma of five healthy controls and five study enrollees (PAH), and d) quantification of densitometry. In PAH patients $1-5$, there was a decrease in plasma nitrotyrosine levels to those of controls by $4 \mathrm{~h}\left({ }^{\#}: \mathrm{p}=0.004\right)$ that was sustained at $24 \mathrm{~h}\left({ }^{\Uparrow}: \mathrm{p}=0.02\right)$. See supplementary figure S1 for full original blot. SOD2: superoxide dismutase 2; PAH: pulmonary arterial hypertension. 
TABLE 2 Effect of GSK2586881 on plasma cytokines

\begin{tabular}{|c|c|c|c|c|c|c|}
\hline & \multirow[t]{2}{*}{ Controls } & \multicolumn{5}{|c|}{ Time after drug administration } \\
\hline & & $\mathbf{O h}$ & $2 \mathrm{~h}$ & $4 \mathrm{~h}$ & $24 \mathrm{~h}$ & 2 weeks \\
\hline IFN- $\gamma \mathrm{pg} \cdot \mathrm{mL}^{-1}$ & $8.4 \pm 5.1$ & $17.1 \pm 10.9$ & $14.9 \pm 12.9$ & $15.4 \pm 9.8^{9}$ & $20.5 \pm 17.8$ & $15.7 \pm 11.2$ \\
\hline $\mathrm{IL}-10 \mathrm{pg} \cdot \mathrm{mL}^{-1}$ & $6.6 \pm 5.5$ & $15.9 \pm 5.3^{\#}$ & $12.0 \pm 3.8^{\text {ा }}$ & $13.8 \pm 6.6$ & $19.1 \pm 10.4$ & $13.6 \pm 9.1$ \\
\hline $\mathrm{IL}-13 \mathrm{pg} \cdot \mathrm{mL}^{-1}$ & $11.6 \pm 9.5$ & $39.1 \pm 15.0^{\#}$ & $30.3 \pm 14.3$ & $32.9 \pm 14.2^{9}$ & $41.0 \pm 22.2$ & $34.9 \pm 18.6$ \\
\hline $\mathrm{IL}-1 \beta \mathrm{pg} \cdot \mathrm{mL}^{-1}$ & $1.3 \pm 0.5$ & $3.8 \pm 2.0^{\#}$ & $2.6 \pm 1.8^{9}$ & $3.0 \pm 1.7^{+}$ & $4.3 \pm 3.2$ & $3.0 \pm 1.8^{\text {वा }}$ \\
\hline $\mathrm{IL}-2 \mathrm{pg} \cdot \mathrm{mL}^{-1}$ & $3.1 \pm 1.4$ & $6.3 \pm 3.0$ & $4.8 \pm 3.0$ & $5.6 \pm 2.8^{\pi}$ & $7.1 \pm 5.2$ & $5.6 \pm 2.6$ \\
\hline $\mathrm{IL}-4 \mathrm{pg} \cdot \mathrm{mL}^{-1}$ & $14.0 \pm 11.4$ & $42.8 \pm 26.5^{\#}$ & $34.5 \pm 23.3$ & $35.7 \pm 19.7$ & $50.0 \pm 35.7$ & $36.9 \pm 27.3$ \\
\hline IL-6 pg:mL & $2.5 \pm 1.8$ & $4.6 \pm 1.8$ & $4.0 \pm 1.9$ & $9.2 \pm 6.7$ & $6.4 \pm 6.4$ & $3.3 \pm 1.5^{+}$ \\
\hline $\mathrm{IL}-8 \mathrm{pg} \cdot \mathrm{mL}^{-1}$ & $5.0 \pm 1.6$ & $8.6 \pm 1.5^{\#}$ & $7.5 \pm 2.5$ & $8.5 \pm 1.6$ & $9.2 \pm 2.9$ & $6.9 \pm 1.4^{9}$ \\
\hline $\mathrm{TNF}-\alpha \mathrm{pg} \cdot \mathrm{mL}^{-1}$ & $4.8 \pm 3.2$ & $9.1 \pm 1.8^{\#}$ & $5.6 \pm 3.0^{9}$ & $6.8^{+} \pm 1.9$ & $9.6 \pm 5.3$ & $5.2 \pm 1.5^{\Uparrow}$ \\
\hline
\end{tabular}

Data are presented as mean \pm SD for five healthy controls and five pulmonary arterial hypertension patients treated with angiotensin-converting enzyme 2. IFN: interferon; IL: interleukin; TNF: tumour necrosis factor. \#: $p<0.05$ versus controls by paired t-test with normalised data; ": $p<0.05$ versus pre-treatment (time-point $0 \mathrm{~h})$ by paired t-test with normalised data; ${ }^{+}: p<0.01$ versus pre-treatment (time-point 0 h) by paired t-test with normalised data.

associated with sustained anti-inflammatory effects with reduced levels of IL-1 $\beta$, IL-6, IL- 8 and TNF- $\alpha$ at 2 weeks $(p<0.05)$. These data collectively suggested a broad suppression of inflammatory cytokines with rhACE2 administration in PAH.

\section{Effect of ACE2 on oxidant stress}

Plasma levels of oxidised lipids are known to be increased in PAH as a marker of oxidant stress $[16,17]$. Isofurans are lipid peroxidation products similar to isoprostanes, but isofurans exhibit favoured formation in the presence of increased oxygen availability [36]. The ratio of isoprostanes to isofurans is a useful index of oxygen availability. We measured isoprostanes and isofurans in the plasma of our trial participants, and found a significant decrease in the isoprostane/isofuran ratio $4 \mathrm{~h}$ after GSK2586881 administration $(\mathrm{p}<0.05)$ (figure $4 \mathrm{~b})$.

To further assess the effect of GSK2586881 on markers of oxidant stress, we quantified plasma 3 -nitrotyrosine levels by dot-blot assay in five healthy controls and in our study participants (figure $4 \mathrm{c}$ and d). We found that nitrotyrosine was increased in PAH patients compared with controls $(p=0.02)$ at baseline. With GSK2586881 administration, plasma nitrotyrosine levels fell in PAH patients at both 4 and $24 \mathrm{~h}(\mathrm{p}=0.004$ and $\mathrm{p}=0.02$, respectively). At the 24-h time-point, $\mathrm{PAH}$ nitrotyrosine levels were no different from controls after GSK2586881 administration, suggesting a significant reduction in whole-body oxidant stress.

\section{Discussion}

Our study sought to 1) demonstrate reduced ACE2 activity in human PAH, 2) define robust readouts of Mas1 agonism in animal models and determine if these readouts are potentially relevant in human PAH, and 3) perform a pilot trial of GSK2586881 in PAH patients to determine if this biological is potentially safe in human PAH and may demonstrate either haemodynamic or biochemical efficacy. We aimed to derive a marker of Mas1 agonism in our pig pulmonary artery model that was of relevance to PAH, and to test the haemodynamic and pharmacodynamics effects of GSK2586881 in human PAH patients. These experiments demonstrate that rhACE2 administration in human PAH may have potential biochemical and haemodynamic efficacy through Mas1 activation. A visual summary of the findings and interpretations is shown in figure 5 .

Given compelling pre-clinical data in rodent models of PAH [18-21] and pathology specimens demonstrating upregulation of the RAS in PAH [3,4], interventions on the RAS pathway would seem likely to have benefit. After demonstrating relevance of this pathway to human PAH and defining inflammatory markers and SOD2 as potential markers of drug effect relevant to pulmonary vascular disease, we proceeded with a pilot study to assess the safety of GSK2586881 in PAH, restricting entry to idiopathic and heritable $\mathrm{PAH}$ as our rodent model experiments were most relevant to these $\mathrm{PAH}$ subgroups [18]. We enrolled five patients with advanced disease, as evidenced by multiple PAH-directed therapies including those targeting the prostacyclin pathway. We did not identify any safety concerns, although we enrolled relatively low numbers of patients. Even in this small study there were potential 


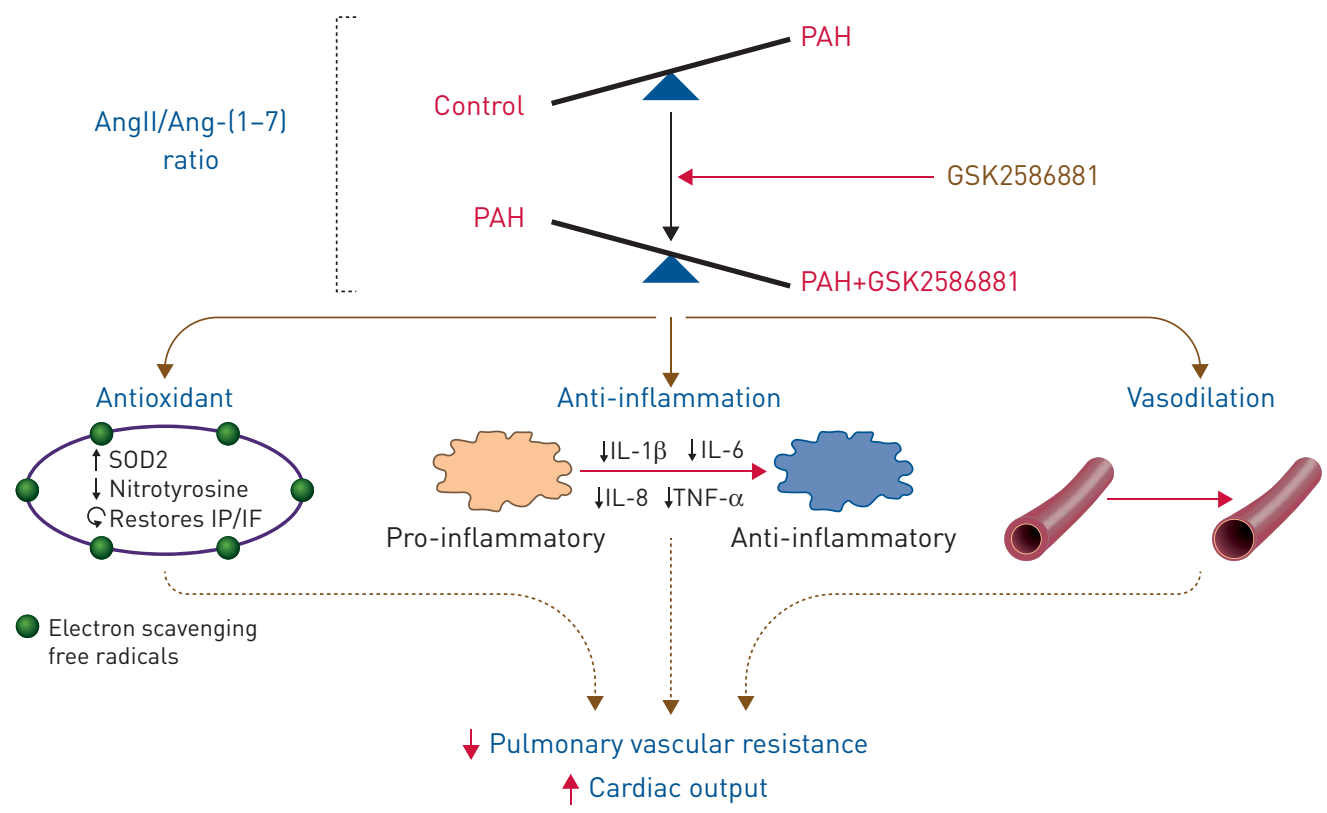

FIGURE 5 Schematic of study findings. PAH: pulmonary arterial hypertension; Angll: angiotensin II; Ang-(1-7): angiotensin-(1-7); SOD2: superoxide dismutase 2; IP: isoprostane; IF: isofuran; IL: interleukin; TNF: tumour necrosis factor.

haemodynamic markers of efficacy, including improved cardiac output and PVR. Furthermore, there was additional biochemical data supporting improved cardiac output: the drop in creatinine may be consistent with improved renal perfusion and the reduced isoprostane/isofuran ratio may indicate improved oxygen delivery to the systemic circulation.

A major hindrance to drug development in PAH is the lack of effective biological markers of drug efficacy. We sought to overcome this limitation by first developing markers of drug effect in relevant tissue, i.e. the pig pulmonary artery. We identified SOD2 and inflammatory pathway genes as having robust changes in response to AVE0991 exposure. SOD2 plays a role in animal models of PAH, although data in human $\mathrm{PAH}$ are limited [32-34]. Using proteomic data, we showed that SOD2 protein was reduced in PAH plasma compared with controls, suggesting potential relevance in humans with detectable protein levels. Human and rodent data show a role for inflammation in PAH $[35,37,38]$, thus we tested cytokine levels in our own PAH population and found elevations in several cytokines in plasma compared with controls. After demonstrating the potential relevance of SOD2 and inflammation in PAH, we next tested the potential effect of Mas1 activation on these markers in human PAH. We found that rhACE2 augmentation resulted in similar effects in the plasma of PAH patients as direct Mas1 activation in pig arteries. These findings correlated with improved cardiac output at the time of maximum suppression of plasma cytokines and maximum reduction in plasma nitrotyrosine levels, a downstream marker of SOD2 activity. SOD2 levels rose at a later time-point in the plasma, but this was expected as SOD2 is located in the mitochondria and cell turnover would be required prior to detection of a change in plasma levels. Although the AngII/Ang-(1-7) ratio also reflected an effective increase in plasma ACE2 activity, these changes in SOD2, nitrotyrosine and cytokines are likely more reflective of activation of Mas1 with changes in downstream signalling. Indeed, prior work has demonstrated that Ang-(1-7) inhibits leukocyte migration and cytokine expression $[39,40]$. While inflammation and SOD2 are potential markers of drug effect, their levels may be altered by other exposures and pathology, making the relative change with drug exposure particularly of interest. It is possible that future drug trials in this pathway may use these markers of Mas1 agonism as early readouts of biochemical efficacy in adaptive trial designs or to define patients most likely to benefit from these interventions.

Oxidant stress has long been implicated in PAH [33, 41-46]. Whole-body markers of oxidant stress include F2-isoprostanes, formed through free radical peroxidation of essential fatty acids, and nitrotyrosine, a product of tyrosine nitration by reactive nitrogen species. Both nitrotyrosine and isoprostanes have been implicated in PAH [17, 44, 47]. Isofurans, in contrast, are nonenzymatic compounds formed through free radical peroxidation of arachidonic acid, and indicate increased production of oxidants at the level of the mitochondria and thus require oxygen to produce. We hypothesised that increased Mas1 signalling would reduce the total burden of oxidant stress in PAH 
patients. We found a reduction in plasma nitrotyrosine by dot-blot assay, consistent with this hypothesis. There was a significant decrease in the plasma isoprostane/isofuran ratio at $4 \mathrm{~h}$ that correlated with the time-point of greatest improvement in cardiac output, suggesting increased delivery of oxygen to tissues and potentially greater oxygen-dependent respiration reflected in reduction in this ratio, although this hypothesis requires further confirmation. Overall, the data were reassuring that there was not an increase, and rather a decrease, in total body oxidant stress as measured by nitrotyrosine levels that showed a reduction to normal levels at 4 and $24 \mathrm{~h}$ after drug administration.

Our study is limited by the low number of trial participants; however, our intention was to perform a proof-of-concept study and to identify potential markers of drug efficacy that can be used in a larger trial. Our RNA expression markers of AVE0991 effects in the pig vasculature were reduced by the known transcriptome of pigs, which is limited compared with humans, and thus there may be more significantly changed transcripts that could not be identified by our methodology; nonetheless, we were able to detect robust readouts. There are other members of the RAS peptide family that would be informative regarding ACE2 activity, including angiotensin-(1-9) and alamandine [48]; however, these compounds rapidly degrade and thus could not be measured in our samples. Also, we did not administer rhACE2 to healthy controls to measure the effect on oxidant stress of inflammatory compounds; however, literature supports comparing an individual's own cytokine panel before and after intervention, rather than comparing groups given the variability in these measurements $[49,50]$.

In conclusion, our data show evidence of reduced ACE2 activity in human PAH accompanied by reduced SOD2 activity, increased cytokine expression and increased oxidant stress. Infusion of a single dose of rhACE2 (GSK2586881) was well tolerated and may have potential haemodynamic benefit. GSK2586881 administration was associated with increased SOD2 levels, reduction in markers of inflammation and reduced plasma oxidant stress. Further study of GSK2586881 as a therapeutic in PAH is warranted.

Acknowledgements: The authors wish to thank Aili L. Lazaar (GlaxoSmithKline, King of Prussia, PA, USA) and Kelly Hardes (GlaxoSmithKline, Stevenage, UK) for their assistance in planning the clinical studies, data analysis and providing review of the manuscript.

Conflict of interest: A.R. Hemnes reports grants from the National Institutes of Health (NIH) and the Cardiovascular Medical Research and Education Fund, personal fees from Actelion, Bayer and United Therapeutics, and personal fees and nonfinancial support (drug supplies to perform experiments) from GlaxoSmithKline, during the conduct of the study. In addition, she has a patent Annamometer issued (noninvasive diagnosis of pulmonary vascular disease). E.A. Austin has received grants from the NIH. He has served as a consultant to Acceleron. E.L. Brittain has received grants from the NIH. He has served as a consultant to Hovine Pharmaceuticals. E.J. Carrier has received grants from the NIH. She has intellectual property pending with Cumberland Pharmaceuticals and has received a grant from this entity. J.P. Fessel has received grant support from the NIH, Gilead, Actelion, the LAM foundation and the Parker B. Francis Foundation. C.D. Fike is a co-inventor on patent applications filed on behalf of Vanderbilt University in the USA and abroad and licensed to Asklepion Pharmaceuticals for the use of intravenous citrulline in lung conditions, without current financial compensation. J.H. Newman has received grants from the NIH. M.E. Pugh has received grant funding from the NIH and has served as a consultant to Gilead. T.W. Rice has received grant funding from the NIH. He has served as a consultant to Avisa Pharma, ACI Clinical and Nestle. He is the Director of Medical Affairs at Cumberland Pharmaceuticals. I.M. Robbins has received funding from the NIH. J.E. Loyd has received grants from the NIH. J. West has received grants from the NIH and Cumberland Pharmaceuticals, and holds intellectual property without financial gain at Cumberland Pharmaceuticals.

Support statement: This work was supported by National Heart, Lung, and Blood Institute grant P01-HL-108800. The project was supported by CTSA award UL1TR000445 from the National Center for Advancing Translational Sciences. Its contents are solely the responsibility of the authors and do not necessarily represent official views of the National Center for Advancing Translational Sciences or the National Institutes of Health. GlaxoSmithKline provided investigational compound and in-kind support in the form of RAS peptide assays. Funding information for this article has been deposited with the Crossref Funder Registry.

\section{References}

1 de Man FS, Tu L, Handoko ML, et al. Dysregulated renin-angiotensin-aldosterone system contributes to pulmonary arterial hypertension. Am J Respir Crit Care Med 2012; 186: 780-789.

2 Morrell NW, Danilov SM, Satyan KB, et al. Right ventricular angiotensin converting enzyme activity and expression is increased during hypoxic pulmonary hypertension. Cardiovasc Res 1997; 34: 393-403.

3 Morrell NW, Atochina EN, Morris KG, et al. Angiotensin converting enzyme expression is increased in small pulmonary arteries of rats with hypoxia-induced pulmonary hypertension. J Clin Invest 1995; 96: 1823-1833.

4 Morrell NW, Morris KG, Stenmark KR. Role of angiotensin-converting enzyme and angiotensin II in development of hypoxic pulmonary hypertension. Am J Physiol 1995; 269: H1186-H1194.

5 Alpert MA, Pressly TA, Mukerji V, et al. Short- and long-term hemodynamic effects of captopril in patients with pulmonary hypertension and selected connective tissue disease. Chest 1992; 102: 1407-1412.

6 Ikram H, Maslowski AH, Nicholls MG, et al. Haemodynamic and hormonal effects of captopril in primary pulmonary hypertension. Br Heart J 1982; 48: 541-545.

7 Galie N, Corris PA, Frost A, et al. Updated treatment algorithm of pulmonary arterial hypertension. J Am Coll Cardiol 2013; 62: D60-D72. 
8 Galie N, Humbert M, Vachiery JL, et al. 2015 ESC/ERS Guidelines for the diagnosis and treatment of pulmonary hypertension. Eur Respir J 2015; 46: 903-975.

9 Lambert DW, Hooper NM, Turner AJ. Angiotensin-converting enzyme 2 and new insights into the reninangiotensin system. Biochem Pharmacol 2008; 75: 781-786.

10 Tallant EA, $\mathrm{Lu} \mathrm{X}$, Weiss RB, et al. Bovine aortic endothelial cells contain an angiotensin-(1-7) receptor. Hypertension 1997; 29: 388-393.

11 Ferrario CM, Chappell MC, Tallant EA, et al. Counterregulatory actions of angiotensin-(1-7). Hypertension 1997; 30: 535-541.

12 Brosnihan KB, Li P, Ferrario CM. Angiotensin-(1-7) dilates canine coronary arteries through kinins and nitric oxide. Hypertension 1996; 27 : 523-528.

13 Nakamoto H, Ferrario CM, Fuller SB, et al. Angiotensin-(1-7) and nitric oxide interaction in renovascular hypertension. Hypertension 1995; 25: 796-802.

14 Zhang Y, Liu J, Luo JY, et al. Upregulation of angiotensin (1-7)-mediated signaling preserves endothelial function through reducing oxidative stress in diabetes. Antioxid Redox Signal 2015; 23: 880-892.

15 Zamanian RT, Hansmann G, Snook S, et al. Insulin resistance in pulmonary arterial hypertension. Eur Respir J 2009; 33: 318-324.

16 Robbins IM, Morrow JD, Christman BW. Oxidant stress but not thromboxane decreases with epoprostenol therapy. Free Radic Biol Med 2005; 38: 568-574.

17 Christman BW, McPherson CD, Newman JH, et al. An imbalance between the excretion of thromboxane and prostacyclin metabolites in pulmonary hypertension. N Engl J Med 1992; 327: 70-75.

18 Johnson JA, Hemnes AR, Perrien DS, et al. Cytoskeletal defects in Bmpr2-associated pulmonary arterial hypertension. Am J Physiol Lung Cell Mol Physiol 2012; 302: L474-L484.

19 Johnson JA, West J, Maynard KB, et al. ACE2 improves right ventricular function in a pressure overload model. PLoS One 2011; 6: e20828.

20 Shenoy V, Gjymishka A, Jarajapu YP, et al. Diminazene attenuates pulmonary hypertension and improves angiogenic progenitor cell functions in experimental models. Am J Respir Crit Care Med 2013; 187: 648-657.

21 Ferreira AJ, Shenoy V, Yamazato Y, et al. Evidence for angiotensin-converting enzyme 2 as a therapeutic target for the prevention of pulmonary hypertension. Am J Respir Crit Care Med 2009; 179: 1048-1054.

22 Shenoy V, Ferreira AJ, Qi Y, et al. The angiotensin-converting enzyme 2/angiogenesis-(1-7)/Mas axis confers cardiopulmonary protection against lung fibrosis and pulmonary hypertension. Am J Respir Crit Care Med 2010; 182: 1065-1072.

23 Breitling S, Krauszman A, Parihar R, et al. Dose-dependent, therapeutic potential of angiotensin-(1-7) for the treatment of pulmonary arterial hypertension. Pulm Circ 2015; 5: 649-657.

24 Liu C, Xiao L, Li F, et al. Generation of outbred Ace2 knockout mice by RNA transfection of TALENs displaying colitis reminiscent pathophysiology and inflammation. Transgenic Res 2015; 24: 433-446.

25 Haschke M, Schuster M, Poglitsch M, et al. Pharmacokinetics and pharmacodynamics of recombinant human angiotensin-converting enzyme 2 in healthy human subjects. Clin Pharmacokinet 2013; 52: 783-792.

26 Fike CD, Aschner JL, Zhang Y, et al. Impaired NO signaling in small pulmonary arteries of chronically hypoxic newborn piglets. Am J Physiol Lung Cell Mol Physiol 2004; 286: L1244-L1254.

27 West JD, Austin ED, Gaskill C, et al. Identification of a common Wnt-associated genetic signature across multiple cell types in pulmonary arterial hypertension. Am J Physiol Cell Physiol 2014; 307: C415-C430.

28 Austin ED, Menon S, Hemnes AR, et al. Idiopathic and heritable PAH perturb common molecular pathways, correlated with increased MSX1 expression. Pulm Circ 2011; 1: 389-398.

29 Simonneau G, Gatzoulis MA, Adatia I, et al. Updated clinical classification of pulmonary hypertension. J Am Coll Cardiol 2013; 62: D34-D41.

30 Barnes PJ, Liu SF. Regulation of pulmonary vascular tone. Pharmacol Rev 1995; 47: 87-131.

31 Sanchez O, Marcos E, Perros F, et al. Role of endothelium-derived CC chemokine ligand 2 in idiopathic pulmonary arterial hypertension. Am J Respir Crit Care Med 2007; 176: 1041-1047.

32 Archer SL, Marsboom G, Kim GH, et al. Epigenetic attenuation of mitochondrial superoxide dismutase 2 in pulmonary arterial hypertension: a basis for excessive cell proliferation and a new therapeutic target. Circulation 2010; 121: 2661-2671.

33 Archer SL, Gomberg-Maitland M, Maitland ML, et al. Mitochondrial metabolism, redox signaling, and fusion: a mitochondria-ROS-HIF- $1 \alpha-\mathrm{Kv} 1.5 \mathrm{O}_{2}$-sensing pathway at the intersection of pulmonary hypertension and cancer. Am J Physiol Heart Circ Physiol 2008; 294: H570-H578.

34 Nozik-Grayck E, Woods C, Taylor JM, et al. Selective depletion of vascular EC-SOD augments chronic hypoxic pulmonary hypertension. Am J Physiol Lung Cell Mol Physiol 2014; 307: L868-L876.

35 Soon E, Holmes AM, Treacy CM, et al. Elevated levels of inflammatory cytokines predict survival in idiopathic and familial pulmonary arterial hypertension. Circulation 2010; 122: 920-927.

36 Roberts LJ 2nd, Fessel JP, Davies SS. The biochemistry of the isoprostane, neuroprostane, and isofuran pathways of lipid peroxidation. Brain Pathol 2005; 15: 143-148.

37 Price LC, Wort SJ, Perros F, et al. Inflammation in pulmonary arterial hypertension. Chest 2012; 141: 210-221.

38 Price LC, Montani D, Tcherakian C, et al. Dexamethasone reverses monocrotaline-induced pulmonary arterial hypertension in rats. Eur Respir J 2011; 37: 813-822.

39 Thomas MC, Pickering RJ, Tsorotes D, et al. Genetic Ace2 deficiency accentuates vascular inflammation and atherosclerosis in the ApoE knockout mouse. Circ Res 2010; 107: 888-897.

40 da Silveira KD, Coelho FM, Vieira AT, et al. Anti-inflammatory effects of the activation of the angiotensin-(1-7) receptor, MAS, in experimental models of arthritis. J Immunol 2010; 185: 5569-5576.

41 Jankov RP, Kantores C, Pan J, et al. Contribution of xanthine oxidase-derived superoxide to chronic hypoxic pulmonary hypertension in neonatal rats. Am J Physiol Lung Cell Mol Physiol 2008; 294: L233-L245.

42 Toporsian M, Jerkic M, Zhou YQ, et al. Spontaneous adult-onset pulmonary arterial hypertension attributable to increased endothelial oxidative stress in a murine model of hereditary hemorrhagic telangiectasia. Arterioscler Thromb Vasc Biol 2010; 30: 509-517.

43 Wood KC, Hsu LL, Gladwin MT. Sickle cell disease vasculopathy: a state of nitric oxide resistance. Free Radic Biol Med 2008; 44: 1506-1528. 
44 Zhao YY, Zhao YD, Mirza MK, et al. Persistent eNOS activation secondary to caveolin-1 deficiency induces pulmonary hypertension in mice and humans through PKG nitration. J Clin Invest 2009; 119: 2009-2018.

45 Giaid A, Saleh D. Reduced expression of endothelial nitric oxide synthase in the lungs of patients with pulmonary hypertension. N Engl J Med 1995; 333: 214-221.

46 Champion HC, Bivalacqua TJ, D'Souza FM, et al. Gene transfer of endothelial nitric oxide synthase to the lung of the mouse in vivo. Effect on agonist-induced and flow-mediated vascular responses. Circ Res 1999; 84: 1422-1432.

47 Bowers R, Cool C, Murphy RC, et al. Oxidative stress in severe pulmonary hypertension. Am J Respir Crit Care Med 2004; 169: 764-769.

48 Jankowski V, Vanholder R, van der Giet M, et al. Mass-spectrometric identification of a novel angiotensin peptide in human plasma. Arterioscler Thromb Vasc Biol 2007; 27: 297-302.

49 Hosnijeh FS, Krop EJ, Portengen L, et al. Stability and reproducibility of simultaneously detected plasma and serum cytokine levels in asymptomatic subjects. Biomarkers 2010; 15: 140-148.

50 Biancotto A, Wank A, Perl S, et al. Baseline levels and temporal stability of 27 multiplexed serum cytokine concentrations in healthy subjects. PLoS One 2013; 8: e76091. 